\title{
Expression levels of the long noncoding RNA steroid receptor activator promote cell proliferation and invasion and predict patient prognosis in human cervical cancer
}

\author{
HEE JUNG KIM ${ }^{1 *}$, LEE KYUNG KIM ${ }^{1 *}$, SAN-HUI LEE ${ }^{1,2^{*}}$, \\ SUN AE PARK $^{1}$, KYUNG JIN EOH ${ }^{1}$ and YOUNG TAE KIM ${ }^{1}$
}

\begin{abstract}
${ }^{1}$ Division of Gynecologic Oncology, Department of Obstetrics and Gynecology, Institute of Women's Life Medical Science, Yonsei University College of Medicine, Seoul $03722 ;{ }^{2}$ Department of Obstetrics and Gynecology, Yonsei University Wonju College of Medicine, Weonju, Gangwon-do 26493, Republic of Korea
\end{abstract}

Received February 7, 2018; Accepted July 26, 2018

DOI: $10.3892 / \mathrm{ol} .2018 .9265$

\begin{abstract}
Long noncoding RNAs (lncRNAs) are involved in developmental processes and diseases and function as critical regulators of a number of different cancer types. Previous research has revealed that IncRNAs affect cervical cancer development. Steroid receptor activator (SRA), an lncRNA, serves as a critical regulator of gynecologic cancer. However, the association between $S R A$ expression and cervical cancer remains unclear. In the present study, the $S R A$ expression levels in patients with cervical cancer were examined and the association between $S R A$ expression and clinicopathological factors was determined. $S R A$ expression was observed in cervical cancer tissues $(\mathrm{n}=100)$ and corresponding normal tissues $(\mathrm{n}=22)$ using reverse transcription-quantitative polymerase chain reaction, and its associations with clinical parameters and prognosis were analyzed. $S R A$ expression was significantly greater in tissues from patients with cervical cancer compared with in control patients $(\mathrm{P}<0.001)$. Multivariate analysis revealed that high $S R A$ expression was an independent prognostic factor of overall survival (hazard ratio $=3.714, \mathrm{P}=0.031$ ). The present study additionally investigated the biofunctional consequences of $S R A$ overexpression in vitro using Cell Counting kit-8, wound healing migration and Matrigel invasion assays. The
\end{abstract}

Correspondence to: Dr Young Tae Kim, Division of Gynecologic Oncology, Department of Obstetrics and Gynecology, Institute of Women's Life Medical Science, Yonsei University College of Medicine, 50-1 Yonsie-ro, Seodaemungu, Seoul 03722, Republic of Korea

E-mail: ytkchoi@yuhs.ac

${ }^{*}$ Contributed equally

Abbreviations: lncRNA, long noncoding RNA; SRA, steroid receptor activator; EMT, epithelial-to-mesenchymal transition

Key words: steroid receptor activator, invasion, metastasis, prognosis, cervical cancer results demonstrated that $S R A$ overexpression enhanced cell proliferation, migration and invasion in vitro. Furthermore, $S R A$ overexpression induced the epithelial-mesenchymal transition (EMT). Therefore, SRA may promote tumor aggressiveness through the upregulation of EMT-associated genes. These results indicated that $S R A$ may represent a novel biomarker for predicting recurrence and prognosis and serve as a promising therapeutic target in cervical cancer.

\section{Introduction}

The most commontypes of malignant tumors in women are breast, uterine, cervical, and ovarian cancers. Cervical cancer is one of the most common types of cancers and is associated with high rates of malignancy-related death in women worldwide. Cervical cancer-related morbidity and mortality rates have decreased over the last 30 years in many countries due to the widespread use of Pap smear screening tests; however, patients with advanced stage cervical cancer still have a poor prognosis (1,2). According to the GLOBOCAN series reported by the International Agency for Research on Cancer, cancers in women have a relatively poor prognosis, with a mortality-to-incidence ratio of $32.5 \%$ (3). In patients with cervical cancer, tumor size, lymph node metastasis, and lymph-blood vessel invasion are independent prognostic factors for survival. However, due to the heterogeneity of the patient population, these factors may not predict prognosis accurately $(4,5)$. Therefore, the identification of novel prognostic biomarkers is urgently needed to improve the prognosis of women with cancer.

Approximately $98 \%$ of transcripts in the human genome represent RNA that does not encode proteins (6). Although these noncoding RNAs (ncRNAs) have previously been considered transcriptional noise, there is now evidence that they play important roles in most cellular processes, including cell proliferation, differentiation, apoptosis, metabolism, and immunity. In addition, ncRNAs affect cancer cell phenotypes and gene regulation $(7,8)$. In recent years, long ncRNAs (lncRNAs) have also been shown to affected DNA binding and the expression of various genes, including chromatin modification complex protein-related activities. Additionally, lncRNAs regulate gene expression in response 
to external stimuli or DNA damage. However, the biological functions and molecular mechanisms of lncRNAs in human diseases and cancers remain largely unknown (9-11).

Steroid receptor activator ( $S R A)$, an IncRNA located on chromosome $5 \mathrm{q} 31.3$, has been shown to activate human hormone receptors that are strongly associated with gynecologic cancers, such as ovarian and breast cancers $(12,13)$. SRA modulates the functions of a variety of transcription factor modulators and can act as a separate scaffold by enhancing the transcriptional activity of the steroid receptor in the reporter gene. SRA is involved in normal biological processes, such as cell death, lipogenesis, steroidogenesis, muscle formation, and insulin signaling, and has been shown to have roles in breast cancer, prostate cancer, abnormal cardiac development, and reduced fertility. Moreover, SRA functionally interacts with proteins involved in cleavage and a number of nuclear receptors, such as retinoic acid receptors (14). Although $S R A$ is known to be related to the progression of malignant tumors, its role in cervical cancer has not been elucidated. Previous studies have shown that SRA expression in cervical cancer cell lines is associated with the progression of malignant tumors (15). However, the clinical relevance of $S R A$ expression is still unclear.

Accordingly, in this study, we investigated the expression of $S R A$ in cervical cancer cell lines and analyzed the relationships among $S R A$ expression, clinicopathological findings, and disease prognosis. Functional analysis was also performed to investigate the effects of $S R A$ on cancer cell invasion and migration in vitro. Finally, we investigated whether $S R A$ was involved in the epithelial-mesenchymal transition (EMT), as a major mechanism leading to metastasis, in cervical cell lines.

\section{Patients and methods}

Patients and tissue samples. In total, 100 women who underwent surgery between 2012 and 2017 at Yonsei Severance Hospital, Yonsei University (Seoul, Korea) were included in this study. Specimens from patients with newly diagnosed invasive stage IA to IVB cervical cancer (International Federation of Gynecology and Obstetrics) who had not received prior treatment were evaluated. Additionally, 22 normal cervical tissues from patients undergoing simple hysterectomy because of uterine leiomyomata were obtained as controls. This study was approved by the Ethics Committee of Yonsei Severance Hospital, and informed consent was obtained from all patients. All specimens were immediately frozen in liquid nitrogen and stored at $-80^{\circ} \mathrm{C}$ until RNA extraction.

Cell lines and cell culture. The human cervical squamous carcinoma SiHa cells was obtained from the Korean Cell Line Bank (Seoul, Korea) and provided by the Korea Gynecologic Cancer Bank through the Bio and Medical Technology Development Program of the Minister of Science, Information and Communication Technology and Future Planning, Korea. A total of 293 cells were purchased from American Type Culture Collection (Manassas, VA, USA). SiHa and 293 cells were cultured in Dulbecco's modified Eagle's medium. All culture media were supplemented with $10 \%$ (v/v) fetal bovine serum and $1 \%$ penicillin/streptomycin, and cell lines were maintained at $37^{\circ} \mathrm{C}$ in a humidified atmosphere of $5 \% \mathrm{CO}_{2}$ and $95 \%$ air. Culture medium was replaced with fresh medium every 2-3 days, and cells that had been passaged less than 20 times were used in the experiments.

Quantitative real-time PCR ( $q R T-P C R)$. Total RNA was extracted using TRIzol reagent (Invitrogen; Thermo Fisher Scientific, Inc., Waltham, MA, USA) according to the manufacturer's instructions. One microgram of total RNA was reverse transcribed into first-strand cDNA using a reverse transcription reagent kit (Bioline, London, UK). The cDNA template was amplified by qRT-PCR using SensiFAST SYBR Hi-ROX Mix (Bioline). qRT-PCR was performed on an ABI StepOnePlus Real-Time PCR System (Applied Biosystems, Foster City, CA, USA). All quantifications were performed with $U 6$ as the internal standard. Relative gene expression was analyzed using the $2^{-\Delta \Delta C T}$ method, and the results were expressed as extent of change with respect to control values. qRT-PCR experiments were replicated at least three times. Primers used for PCR are shown in Table I.

Plasmid constructs and generation of stable cell lines. Full-length human SRA cDNA was amplified by PCR and inserted into the pLenti6/V5-D-TOPO vector using the ViraPower Lentiviral Expression System (Invitrogen; Thermo Fisher Scientific, Inc.) according to the manufacturer's protocol. The plasmid was transfected into 293FT cells for packaging, and the resulting lentivirus was used to infect the desired cell lines. The selection of SRA stably transfected cells was performed in medium containing blasticidin (Invitrogen; Thermo Fisher Scientific, Inc.).

Cell proliferation assay. Cell proliferation was evaluated using Cell Counting kit-8 (CCK-8) assays (Dojindo Laboratories, Kumamoto, Japan). Cells were seeded into 6-well flat-bottomed plates $\left(1 \times 10^{5}\right.$ cells/well $)$ in $2 \mathrm{ml}$ complete medium. The cells were incubated overnight to allow for cell attachment and recovery and were subsequently subjected to $S R A l$ overexpression for 24, 48, 72, or $96 \mathrm{~h}$. Next, $10 \mu \mathrm{l}$ CCK-8 solution was added to each well, and cells were incubated for $1 \mathrm{~h}$. The absorbance was measured at $450 \mathrm{~nm}$ using an auto-microplate reader to calculate the number of viable cells in each well. The cell survival rate was expressed as the absorbance relative to that of the $\mathrm{SiHa}$, veoctor cells. Three independent experiments were performed in triplicate.

Matrigel invasion assay. Matrigel invasion assays were performed using BD Biocoat Matrigel Invasion Chambers (pore size: $8 \mu \mathrm{m}$, 24-well; BD Biosciences, Bedford, MA, USA), according to the manufacturer's protocol. Briefly, $1 \times 10^{5}$ cells were plated in the upper chambers in serum-free medium, and complete medium was added to the bottom chamber. The chambers were then incubated for $48 \mathrm{~h}$ at $37^{\circ} \mathrm{C}$ in an atmosphere containing $5 \% \mathrm{CO}_{2}$. Noninvading cells were removed from the upper chambers using cotton-tipped swabs. Cells that had invaded through the pores into the lower side of the filter were stained (Diff Quik; Sysmes, Kobe, Japan), and these cells were then counted using a hemocytometer. Invasion cell was measured using ImageJ software (NIH, Bethesda, MD, USA) and the percentage of the invasion cell was calculated. Results were standardized to $\mathrm{SiHa}$ cells. The number of cells that invaded the membrane was counted in 10 fields under the $\mathrm{x} 20$ objective lens. Original magnification, $\mathrm{x} 200$. The assay was replicated at least three times. 
Table I. Primer sequences used in this study.

\begin{tabular}{lllr}
\hline & \multicolumn{2}{c}{ Primer sequence } & Product \\
\cline { 2 - 3 } Gene & \multicolumn{1}{c}{ Forward (5'-3') } & Reverse (5'-3') & Size (base pair) \\
\hline SRA & CTCCCTTCTTACCACCACCA & TGCAGATACACAGGGAGCAG & 217 \\
U6 & CTCGCTTCGGCAGCACA & AACGCTTCAGGAATTTGCGT & 92 \\
E-cadherin & ATTCTGATTCTGCTGCTCTTG & AGTAGTCATAGTCCTGGTCCT & 421 \\
N-cadherin & CCCAAGACAAAGAGACCCAG & GCCACTGTGCTTACTGAATTG & 140 \\
Snail & GAGGCGGTGGCAGACTAG & GACACATCGGTCAGACCAG & 178 \\
Wnt & TGTGAGGTGAAGACCTGCTG & AAAGTTGGGGGAGTTCTCGT & 207 \\
Vimentin & TGGATTCACTCCCTCTGGTT & GGTCATCGTGATGCTGAGAA & 111 \\
Twist & CGGGAGTCCGCAGTCTTA & TGAATCTTGCTCAGCTTGTC & 150 \\
\hline
\end{tabular}

SRA, steroid receptor activator.

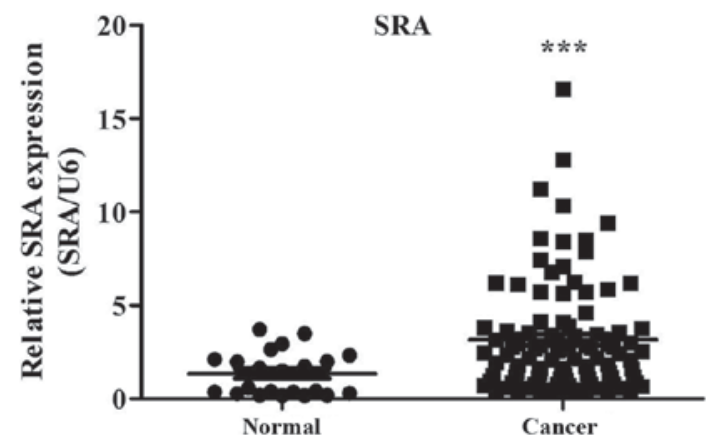

Figure 1. Elevated expression of $S R A$ in cervical cancer tissues. $S R A$ expression was evaluated in cervical cancer tissues $(n=100)$ and normal tissues $(n=22)$ reverse transcription-quantitative polymerase chain reaction with U6 as an internal control. Data are expressed as the mean \pm standard deviation. ${ }^{* * * *} \mathrm{P}<0.0001$ vs. normal control. SRA, steroid receptor activator.

Wound healing migration assay. Cell migration was assessed using wound healing assays. Briefly, $1 \times 10^{6}$ cells were seeded into 6-well culture plates with serum-containing medium and allowed to grow to $90 \%$ confluence in complete medium. The serum-containing medium was removed, and cells were serum starved for $24 \mathrm{~h}$. When the cell density reached $\sim 100 \%$ confluence, an artificial homogenous wound was created by scratching the monolayer with a sterile $200-\mu$ l pipette tip. After scratching, the cells were washed with serum-free medium. Images of cells migrating into the wound were captured at 0,24, and $48 \mathrm{~h}$ using a microscope. Scratch width was measured using NIH ImageJ software, and the percentage of the scratch area closed was calculated as (width at $0 \mathrm{~h}$-width at $48 \mathrm{~h}$ )/width at $0 \mathrm{~h}$. Results were standardized to SiHa cells. The migration cells was counted in 10 fields under the x 20 objective lens. Original magnification, $\mathrm{x} 200$. The assay was performed in triplicate.

Western blot analysis. Proteins were extracted with RIPA buffer (Thermo Fisher Scientific, Inc.). Protein concentrations were measured using a Pierce BCA Protein assay kit (Thermo Fisher Scientific,Inc.). After boiling with $5 \mathrm{X}$ sample buffer, proteins were resolved on $10 \%$ sodium dodecyl sulfate-polyacrylamide gels and transferred electrophoretically to polyvinylidene difluoride membranes (Millipore, Billerica, MA, USA). Membranes were blocked with $5 \%$ nonfat dried milk in $1 \mathrm{X}$ Tris-buffered saline containing $0.1 \%$ Tween-20 (pH 7.6) at room temperature for $1 \mathrm{~h}$ and were then incubated with primary antibodies at $4^{\circ} \mathrm{C}$ overnight under constant agitation. The primary antibodies included rabbit anti-human E-cadherin (1:1,000 dilution), rabbit anti-human $\beta$-catenin (1:1,000 dilution), and mouse anti-human Snail (1:1,000 dilution; all Cell Signaling Technology, Danvers, MA, USA). Proteins were visualized using an enhanced chemiluminescence system (Amersham, Little Chalfont, UK), and band intensities were quantified using a Luminescent Image Analyzer (LAS-4000 mini; Fujifilm, Uppsala, Sweden).

Statistical analysis. The results of the statistical analyses (SPSS, version 24.0; IBM, Chicago, IL, USA) were expressed as means \pm standard deviations (SDs). Pearson's $\chi^{2}$ tests, Student's t-tests, and Fisher's exact tests were used to evaluate the associations between SRA expression and clinicopathological characteristics. To evaluate the performance of the models with respect to their discrimination ability, statistics were used chi-square values from the logrank test from the receiver operating characteristic (ROC) analysis. The median value (1.64) was set as the cut-off value. The groups were classification into high and low SRA expression groups at values above and below the cut-off value (1.64), respectively. The Kaplan-Meier method was used to analyze overall survival times. The log-rank test was used to estimate between group differences. Stepwise Cox regression model analysis was used for multivariate survival analysis of the parameters that were significant in the univariate analysis. The statistical tests were two-sided; differences with $\mathrm{P}<0.05$ was considered to indicate a statistically significant difference.

\section{Results}

SRA levels were elevated in patients with cervical cancer having poor prognoses. To determine whether SRA expression in tissues was linked to the clinicopathological features of cervical cancer, we evaluated the expression of $S R A$ in cervical cancer tissues $(n=100)$ and corresponding normal tissues $(n=22)$. SRA expression in cervical cancer tissues was more than 3.15-fold that of noncancerous tissues 
$(\mathrm{P}=0.00007$; Fig. 1). Additionally, we examined the relationships between SRA expression and clinicopathological information in 100 patients with cervical cancer (Table II). The mean follow-up period was 60 months. Additionally, patients with high SRA expression exhibited higher rates of lymphatic invasion and invasive cell type relative to patients with low SRA expression; this relationship was statistically significant.

The median 5-year survival duration was significantly higher in the low $S R A$ expression group than in the high SRA expression group (19.7 and 25.1 months, respectively; log-rank test: $\mathrm{P}=0.041$; Fig. $2 \mathrm{~A})$. The risk model in SRA data had an area under curve (AUC) of 0.737 in predicting $(\mathrm{P}=0.001$; Fig. 2B). Moreover, Cox univariate proportional hazards analysis showed that stage [hazard ratio $(\mathrm{HR})=2.809$; $\mathrm{P}=0.004]$, tumor size $(\mathrm{HR}=2.260 ; \mathrm{P}=0.011)$, and recurrence $(\mathrm{HR}=2.479 ; \mathrm{P}=0.05)$ were independent prognostic factors for overall survival (Table II). Cox multivariate proportional hazards analysis showed that $S R A$ expression $(\mathrm{HR}=3.714$; $\mathrm{P}=0.031)$, stage $(\mathrm{HR}=2.809 ; \mathrm{P}=0.004)$, tumor size $(\mathrm{HR}=2.143$; $\mathrm{P}=0.047)$, and lymphatic invasion $(\mathrm{HR}=3.44, \mathrm{P}=0.03)$ were independent prognostic factors for overall survival (Table III). Both univariate and multivariate proportional hazards analyses showed that stage and tumor size were independent prognostic factors of overall survival.

Overexpression of SRA increased cell proliferation in cervical cancer cells. We previously examined SRA expression levels in several cervical cancer cell lines by qRT-PCR (15). Here, lentiviral-mediated overexpression of $S R A$ was performed to determine the functional role of this lncRNA in SiHa cells. SRA-expressing plasmid was prepared using as template a PCR product that contained the SRA sequences (Fig. 3A). RT-PCR analysis showed that $S R A$ was successfully overexpressed in SiHa cells compared with that in control cells $(\mathrm{P}=0.01$; Fig. 3B). We next examined the impact of $S R A$ overexpression on cell proliferation. The results of CCK- 8 assays showed that overexpression $S R A$ in $\mathrm{SiHa}$ cells increased cell proliferation (Fig. 3C), suggesting that SRA was involved in the proliferation of cervical cancer cells.

SRA overexpression affected cervical cancer cell migration and invasion. Next, the effects of SRA on the invasive and migratory behaviors of cells were assessed by Matrigel invasion and wound healing assays. Overexpression of SRA resulted in increased migration of $\mathrm{SiHa}$ cells relative to empty vector-expressing controls $(\mathrm{P}=0.001$; Fig. $4 \mathrm{~A})$. There was a significant difference between the scratch width percentages of each cell line 24 and $48 \mathrm{~h}$ after in the SiHa, empty vector and SRA overexpression cells ( $\mathrm{P}=0.001$; Fig. 4B). Furthermore, SRA overexpression in SiHa cells significantly increased invasion relative to that in empty vector-expressing cells $(\mathrm{P}=0.001$; Fig. 4C). The invasion relative percentages of each cell line $48 \mathrm{~h}$ after in SiHa, empty vector and SRA overexpression groups was significantly different $(\mathrm{P}=0.001$; Fig. 4D). The SiHa cells percentages were $100 \pm 4.58$. The empty cell line percentages were $72 \pm 13.52$. The SRA overexpression cell line percentages were $173 \pm 5.38$. Altogether, these results indicated that SRA promoted SiHa cell invasion and migration in vitro.
OverexpressionSRAincreased the expression ofEMT-associated genes in cervical cancer cells. Because the EMT is important in cell migration and invasion, the present study examined whether SRA was required for EMT using RT-qPCR and western blotting. The overexpression of SRA decreased E-cadherin expression and increased N-cadherin, Snail, Wnt5 $\beta$, Vimentin and $\beta$-catenin expression (Fig. 5A). Levels of protein in cells with overexpressed SRA had decreased E-cadherin expression and increased N-cadherin, $\beta$-catenin, Vimentin, Wnt5 $\beta$, Twist and Snail expression (Fig. 5B). The western blot density percentages of each cell line $48 \mathrm{~h}$ after in the SiHa, empty vector and SRA overexpression cells are presented in Fig. 5C. In addition, the expression of Snail, a transcription factor that mediates the EMT, was upregulated in SRA-overexpressing $\mathrm{SiHa}$ cells compared with that in cells transfected with the empty vector (Fig. 5A-C). These data suggested that upregulation of EMT-associated genes could partly explain the involvement of SRA in cervical cancer cell migration and invasion.

\section{Discussion}

A deeper understanding of the molecular mechanisms underlying cervical cancer progression and metastasis is essential for the development of more effective therapeutic treatments and for identifying new diagnostic markers for cervical cancer. lncRNAs are transcripts measuring 200 nucleotides or more that do not encode proteins. Although the functional roles of small regulatory ncRNAs, such as microRNAs, have been well established in human cancers, little is known about the regulatory roles of lncRNAs and their relevance to human disease. Many lncRNAs are capped, spliced, and polyadenylated with protein-coding counterparts (16). IncRNAs have been shown to exhibit tissue-specific expression patterns and have been functionally characterized; the biosynthesis of these RNAs is important for a variety of physiological processes, and abnormal expression of lncRNAs may affect cancer development and progression (17). Moreover, lncRNAs are emerging as key players in the complex mechanisms underlying malignant processes, including tumorigenesis, drug resistance, and metastasis (18-20).

Cervical cancer remains one of the leading causes of cancer-related death in women worldwide (21). Despite the development of advanced therapeutic strategies, the prognosis in patients with cervical cancer varies significantly and is hard to predict. Treatment outcomes still depend primarily on early detection and diagnosis. Recent studies have demonstrated that some abnormal molecular biology changes may play central roles in the development of cervical cancer (22). However, there are few reports of the biology and function of $S R A$ in cervical cancer cells. Accordingly, in this study, investigated the molecular function and clinical significance of $S R A$ expression in cervical cancer cell lines. We found that SRA expression was higher in cervical cancer tissues than in comparable noncancerous tissues. Moreover, $S R A$ overexpression altered cell growth, migration, and invasion in cervical cancer cells. The metastatic effects of $S R A$ appeared to be mediated, at least in part, by the regulation of genes involved in cell migration, invasion, and the EMT.

$S R A$ is an lncRNA that acts as a putative coactivator for steroid receptor-mediated transcription. Its overexpression and consequent deregulated hormone signaling are associated with 
Table II. Clinicopathological features and SRA expression in patients with cervical cancer.

\begin{tabular}{|c|c|c|c|c|}
\hline \multirow[b]{2}{*}{ Variables } & \multirow[b]{2}{*}{$\mathrm{n}(\%)$} & \multicolumn{2}{|c|}{$S R A$ expression } & \multirow[b]{2}{*}{ P-value ${ }^{a}$} \\
\hline & & Low & High & \\
\hline Age (years, mean $\pm S D$ ) & 100 & $49.425 \pm 1.84$ & $51.95 \pm 1.61$ & 0.339 \\
\hline Stage & & & & 0.094 \\
\hline I, II & 64 & 22 & 42 & \\
\hline III, IV & 36 & 18 & 18 & \\
\hline Lymph node metastasis & & & & 0.414 \\
\hline Yes & 35 & 15 & 20 & \\
\hline No & 65 & 25 & 40 & \\
\hline Lymphatic invasion & & & & 0.033 \\
\hline Yes & 37 & 10 & 27 & \\
\hline No & 63 & 30 & 33 & \\
\hline Tumor size $(\mathrm{cm})$ & & & & 0.478 \\
\hline $0-5.9$ & 45 & 19 & 29 & \\
\hline$\geq 6$ & 53 & 21 & 32 & \\
\hline Recurrence & & & & 0.515 \\
\hline Yes & 29 & 12 & 17 & \\
\hline No & 71 & 28 & 43 & \\
\hline Cell type & & & & 0.025 \\
\hline Squamous cell carcinoma & 63 & 20 & 43 & \\
\hline Adenocarcinoma & 17 & 9 & 8 & \\
\hline Mixed & 8 & 3 & 5 & \\
\hline Other & 10 & 7 & 3 & \\
\hline
\end{tabular}

${ }^{a}$ Chi-square test or Fisher's exact test were used to calculate P-values. SRA, steroid receptor activator; SD, standard deviation.
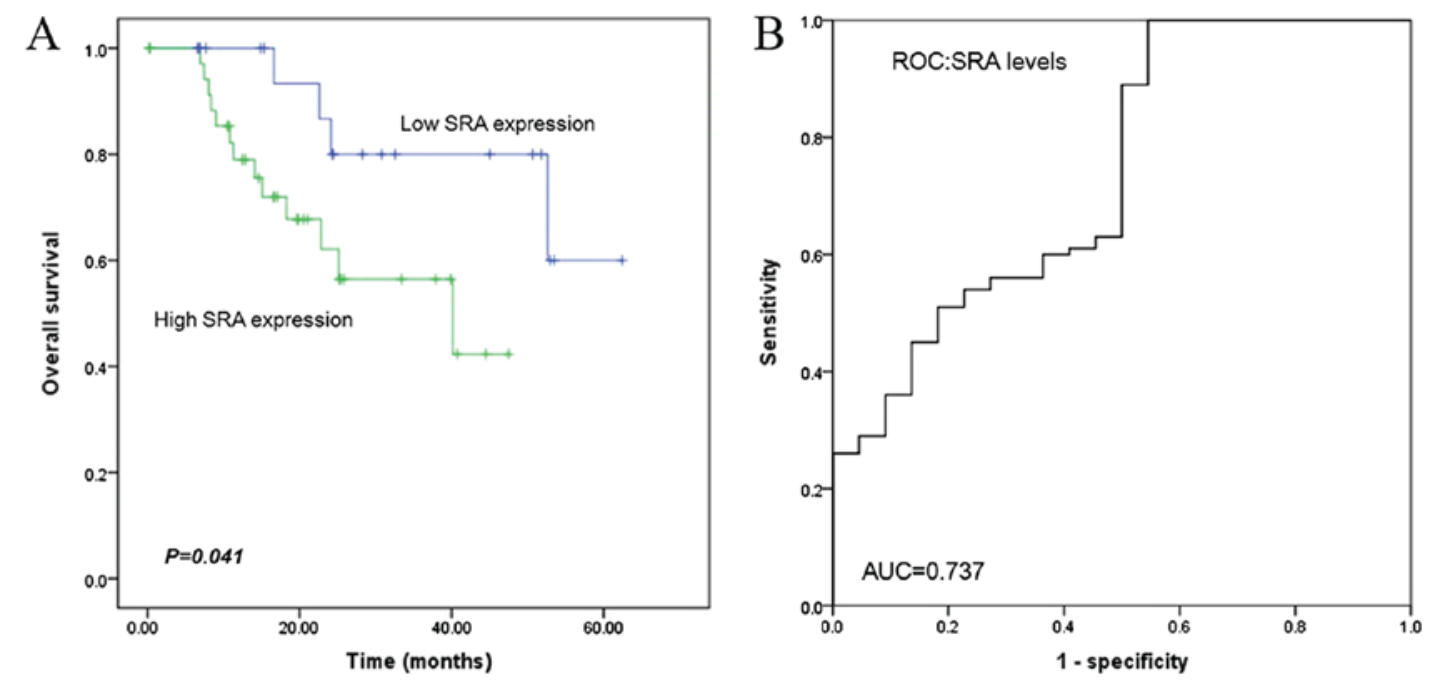

Figure 2. (A) Overall survival of patients in the high SRA group demonstrated significantly worse survival rates than those who were in the low SRA group ( $\mathrm{P}=0.041)$. (B) ROC curve for prognosis prediction of patients using $S R A$ level. The AUC is shown in plots. ROC, receiver operating characteristic; AUC, area under curve; SRA, steroid receptor activator.

breast, uterine, ovarian, and prostate cancers (23). Therefore, we hypothesized that expression of $S R A$ may affect prognosis in patients with cervical cancer. We discovered that $S R A$ overexpression increased cervical cancer cell proliferation, migration, and invasion. Thus, SRA may be oncogenic in cervical cancer and promote aggressive and metastatic characteristics. The EMT involves alterations in cell phenotype, and several transcription factors have been implicated in the regulation of EMT-related gene expression. Although several studies have focused on transcriptional regulators in the pathological EMT, 
Table III. Univariate and multivariate analyses of parameters associated with overall survival in 100 patients with cervical cancer.

\begin{tabular}{llllll}
\hline & \multicolumn{2}{c}{ Univariate analysis } & & \multicolumn{2}{c}{ Multivariate analysis } \\
\cline { 2 - 3 } Factor & Hazard ratio (95\% CI) & P-value & & Hazard ratio (95\% CI) & P-value \\
\hline SRA expression & $2.096(0.747-5.729)$ & 0.162 & & $3.714(1.128-12.230)$ & 0.031 \\
Age (years) & $1.014(0.976-1.052)$ & 0.483 & & $1.023(0.983-1.064)$ & 0.27 \\
Stage & $2.265(1.413-3.631)$ & 0.001 & & $2.809(1.388-5.686)$ & 0.004 \\
Tumor size & $2.260(1.208-4.231)$ & 0.011 & & $2.143(1.011-4.542)$ & 0.047 \\
Lymph node metastasis & $2.252(0.907-5.588)$ & 0.08 & & $0.751(0.240-2.350)$ & 0.623 \\
Lymphovascular invasion & $1.407(0.564-3.507)$ & 0.464 & & $3.44(1.13-10.468)$ & 0.03 \\
Recurrence & $2.479(1.001-6.136)$ & 0.05 & & $2.68(0.734-9.782)$ & 0.136 \\
Histology & $1.185(0.765-1.837)$ & 0.447 & & $1.434(0.883-2.330)$ & 0.145 \\
\hline
\end{tabular}

$S R A$, steroid receptor activator.
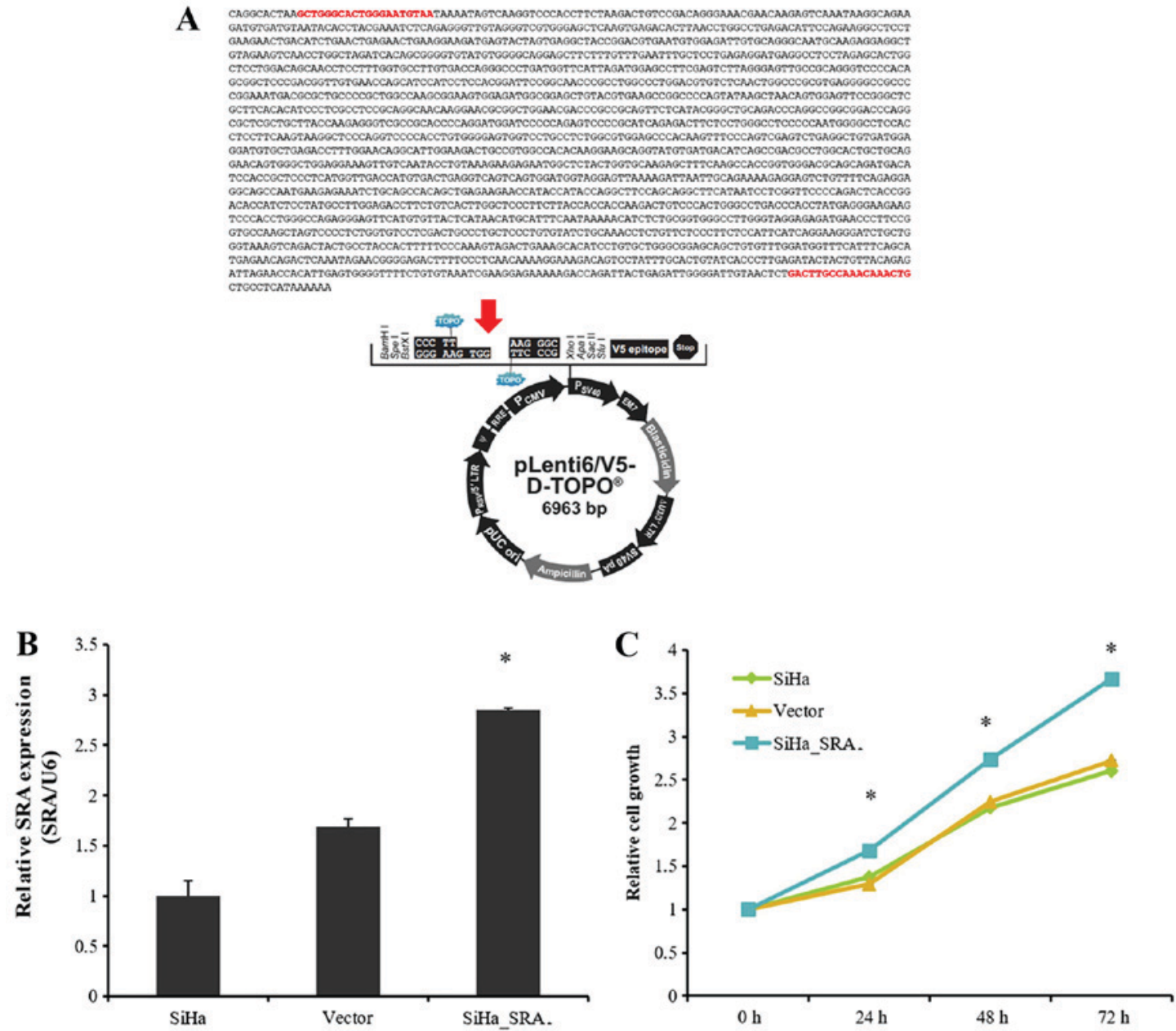

Figure 3. SRA promoted cell proliferation in SiHa cells. (A) Map of plasmid pLenti6/V5-D-TOPO and the sequence of the overexpression fusion gene SRA (B) Overexpression of $S R A$ in SiHa cells was analyzed using reverse transcription-quantitative polymerase chain reaction. (C) Cell proliferation was analyzed using Cell Counting kit- 8 assays. Bars indicate the mean \pm standard deviation of three independent experiments. ${ }^{*} \mathrm{P}<0.05$ vs. SiHa, Vector cells. $\mathrm{SD}$, standard deviation; SRA, steroid receptor activator.

few studies have evaluated the roles of transcription factors in cervical cancer (24).

In this study, high expression of $S R A$ in cervical cancer cells induced cell migration and invasion through upregulation of EMT-related genes.
Loss of E-cadherin is thought to be an important event of EMT, but N-cadherin causes a decrease in intercellular junctions between two adjacent endotheliums, which causes cancer cells to slip (25). In addition, $\beta$-catenin is more mobile and weakens the slowly related mesenchymal phenotype. 
A

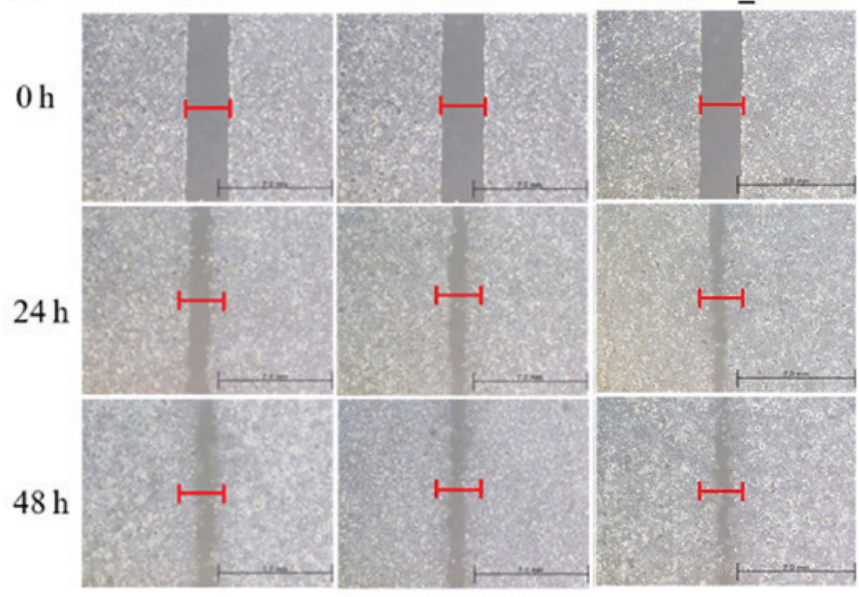

B

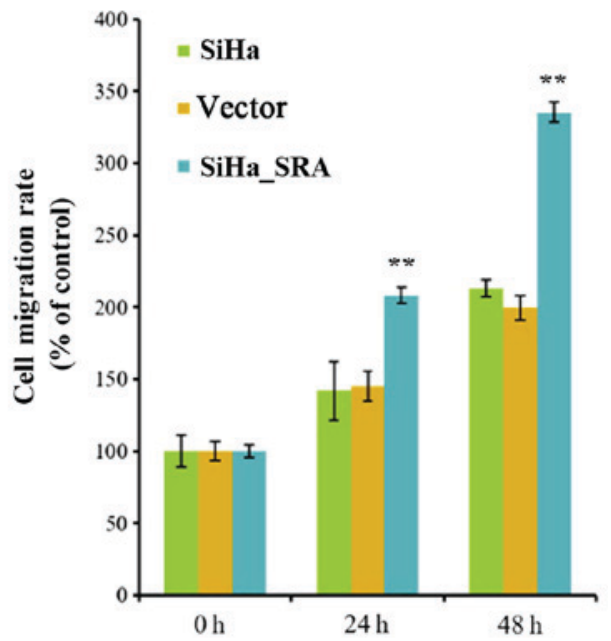

C $\quad \mathrm{SiHa}$

Vector

SiHa_SRA
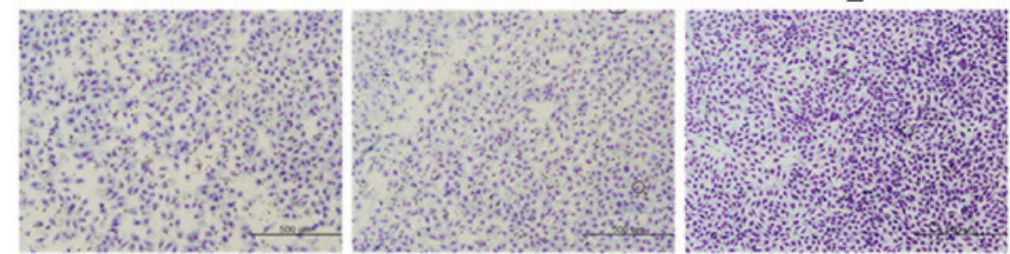

D

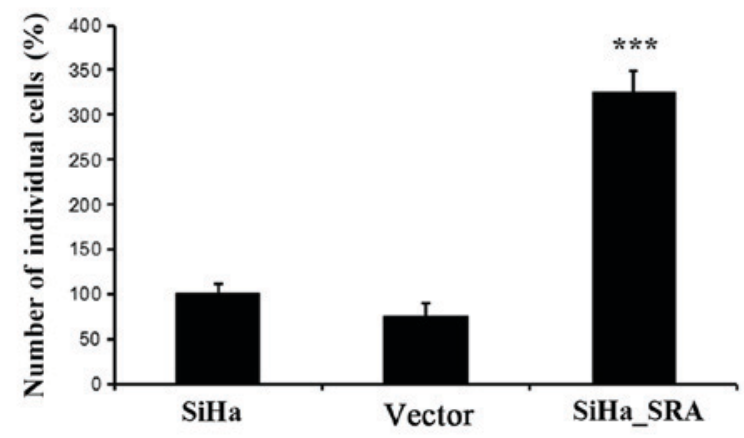

Figure 4. SRA promoted cell migration and invasion. (A) Wound healing assay observed under the optical microscope were used to determine migration in SRA-overexpressing SiHa cells (x200). Cells after 24 and $48 \mathrm{~h}$ analyzed and determine for SiHa cells as a control. (B) Presents the wound healing assay percentages of each cell line. (C) Cell invasion observed under the optical microscope. Matrigel invasion assays were used to determine invasion after $48 \mathrm{~h}$ in SRA-overexpressing SiHa cells. (D) Presents the Matrigel invasion assay percentages of each cell line. Each assay was performed in triplicate. Data are means \pm standard deviation. ${ }^{* *} \mathrm{P}<0.01$ vs. SiHa, Vector cells. ${ }^{* * *} \mathrm{P}<0.0001$ vs. SiHa, Vector cells. SRA, steroid receptor activator.

The enhancement of the expression of transcription factors such as Snail and Twist is associated with the loss of intercellular adhesion (26), vimentin constitutes the main component of the cytoskeleton of mesenchymal cells, it's up-regulation is induced by EMT $(1,27)$. Recent study have been demonstrated that HOTAIR regulated the expression of vascular endothelial growth factor, matrix metalloproteinase-9 and EMT-related genes, which are important for cell motility and metastasis (19).

We hypothesized that $S R A$ may act an important regulator of several signaling mechanisms associated with the EMT. Our results suggested that $S R A$ may contribute to the growth, invasion, and recurrence of cervical cancer through induction of the EMT. The recurrence rate of radical cervical cancer after radical surgery is $15-30 \%$, and the prognosis of patients with recurrence is poor (28). A reliable predictor of recurrence and progression is needed to improve the prognosis of patients with cervical cancer. Cell type in cervical cancer is related to patient survival, and squamous cell carcinoma has been found to be most closely related to survival $(29,30)$. Here, we demonstrated that high $S R A$ expression was related to low overall survival rates in patients with squamous cell carcinoma. Multi-scale modelling Pelvic lymph node metastasis is one of the most important postoperative risk factors for relapse or failure to survive. Therefore, patients with cervical cancer with metastasis to pelvic lymph nodes require adjuvant therapy, such as postoperative radiation therapy (31). We also found that high $S R A$ expression was related to advanced stage and metastasis. Thus, assessment of $S R A$ expression in patients with cervical cancer can inform treatment decisions by predicting the risk of progression or recurrence.

In summary, we found that patients with cervical cancer had elevated SRA levels. Moreover, SRA overexpression was positively correlated with clinicopathological parameters in cervical cancer, and $S R A$ was found to have a role in 
A
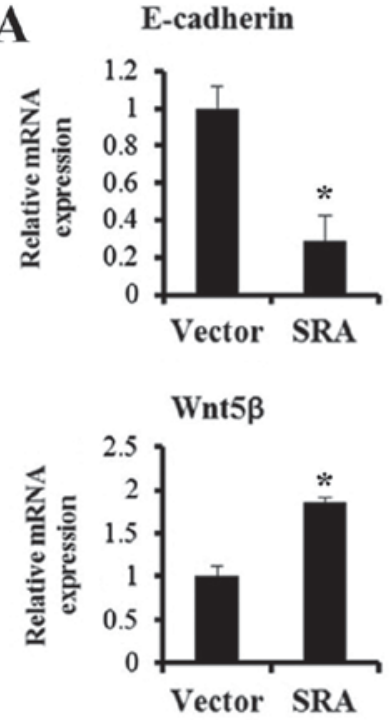

N-cadherin

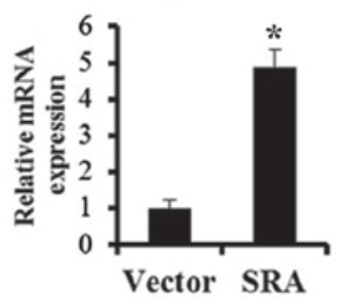

Vimentin

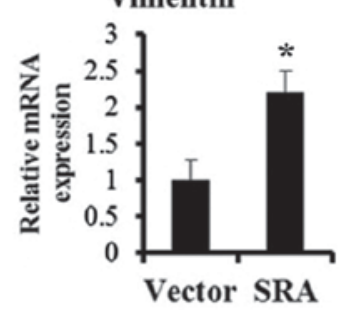

Snail

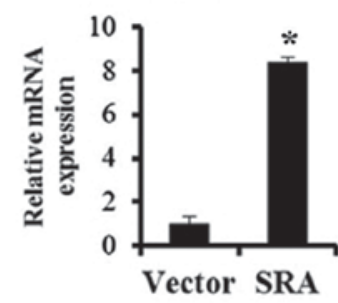

Twist

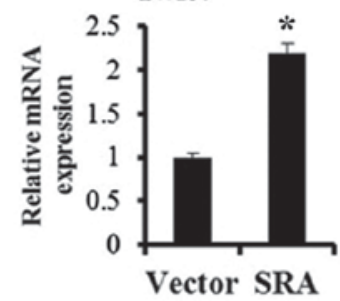

$\beta$-catenin

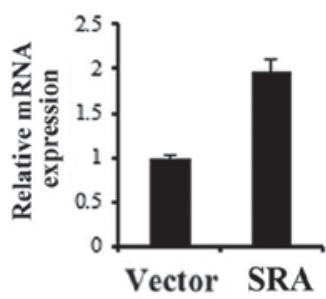

B

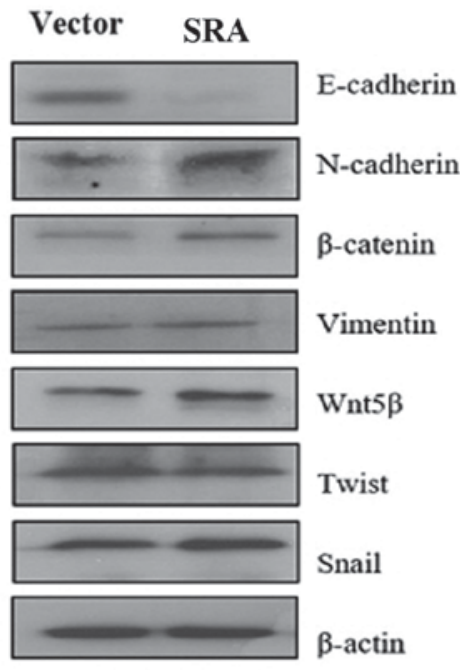

C
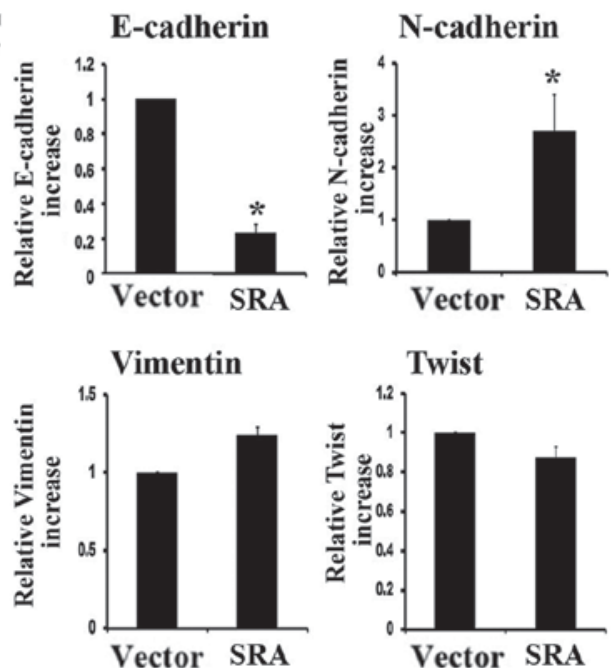
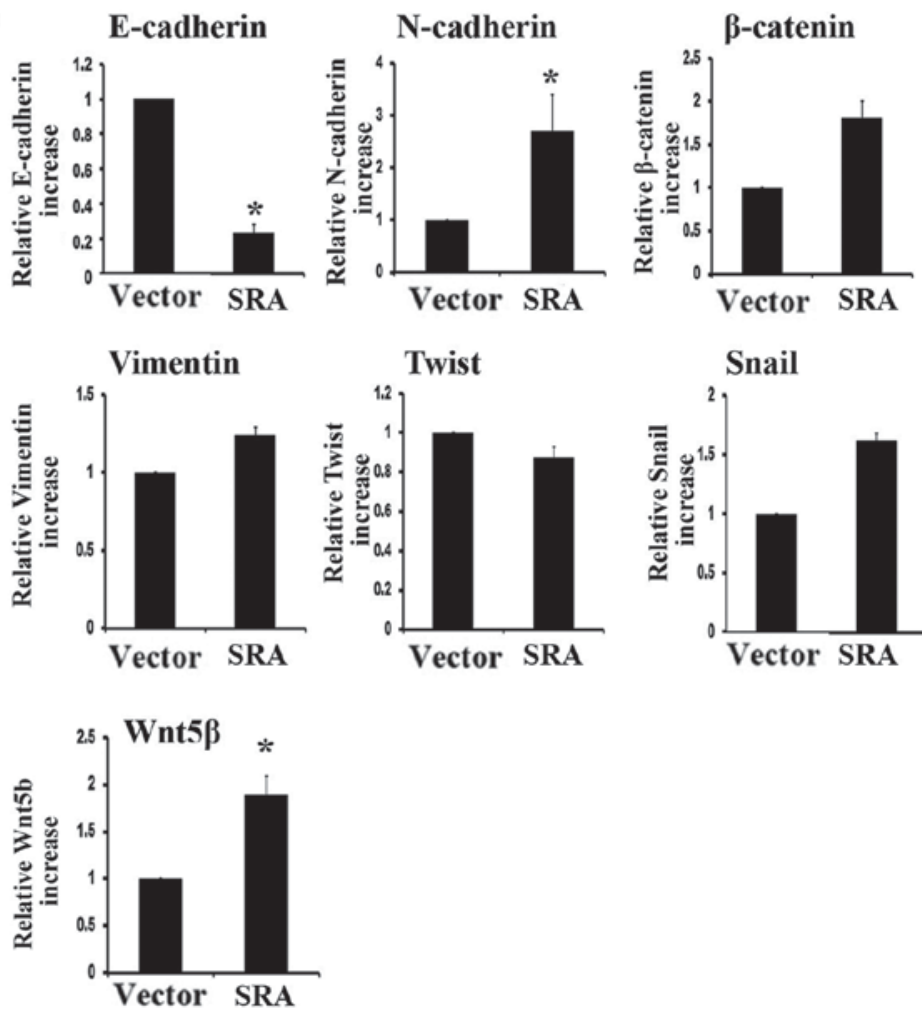

Figure 5. Effects of overexpression SRA on EMT-associated genes in SiHa cells. (A) Levels of E-cadherin, N-cadherin, Snail, $\beta$-catenin, Wnt $5 \beta$, Vimentin and Twist were analyzed by reverse transcription-quantitative polymerase chain reaction in SRA overexpression SiHa cells. (B) Protein lysates were obtained from $S R A$-overexpressing SiHa cells. Levels of proteins in EMT-associated gene were analyzed using western blotting. (C) The band intensities were quantitated. The histogram revealed the average volume density corrected for the loading control ( $\beta$-actin). "P<0.05 vs. SiHa cells. SRA, steroid receptor activator; EMT, epithelial-mesenchymal transition.

promoting cell growth and invasion through modulation of the EMT. These results suggested that SRA may have applications in determination of the clinicopathological stage and/or prognosis of patients with cervical cancer. Accordingly, SRA may be a promising therapeutic target in cervical cancer.

\section{Acknowledgements}

Not applicable.

\section{Funding}

The present study was supported by the Korea Health Technology R\&D Project through the Korea Health Industry Development Institute, funded by the Ministry of Health and Welfare, Republic of Korea (grant no. HI17C0321) and by the Basic Science Research Program through the National Research Foundation of Korea funded by the Ministry of Education, Science and Technology (grant nos. NRF-2015R1A2A2A01008162, 
NRF-2017R1D1A3B03032983 and NRF-2015 R1C1A2A01053516).

\section{Availability of data and materials}

The datasets used/or analyzed during the current study are available from the corresponding author on reasonable request.

\section{Authors' contributions}

HJK, SHL and YTK designed and coordinated the study and LKK wrote the paper. LKK, KJE and SAP performed the research and analyzed the data.

\section{Ethics approval and consent to participate}

The present study was approved by the Ethics Committee of Yonsei Severance Hospital, and written informed consent was obtained from all patients.

\section{Patient consent for publication}

Written informed consent was obtained from all patients.

\section{Competing interests}

The authors declare that they have no competing interests.

\section{References}

1. Ferlay J, Soerjomataram I, Dikshit R, Eser S, Mathers C, Rebelo M, Parkin DM, Forman D and Bray F: Cancer incidence and mortality worldwide: Sources, methods and major patterns in GLOBOCAN 2012. Int J Cancer 136: E359-E386, 2015.

2. Yang W, Hong L, Xu X, Wang Q, Huang J and Jiang L: LncRNA GAS5 suppresses the tumorigenesis of cervical cancer by downregulating miR-196a and miR-205. Tumour Biol 39: $1010428317711315,2017$.

3. Dryden-Peterson S, Bvochora-Nsingo M, Suneja G, Efstathiou JA, Grover S, Chiyapo S, Ramogola-Masire D, Kebabonye-Pusoentsi M, Clayman R, Mapes AC, et al: HIV infection and survival among women with cervical cancer. J Clin Oncol 34: 3749-3757, 2016.

4. Chuang LT, Feldman S, Nakisige C, Temin S and Berek JS: Management and care of women with invasive cervical cancer. J Clin Oncol 34: 3354-3355, 2016.

5. Perez DS, Hoage TR, Pritchett JR, Ducharme-Smith AL, Halling ML, Ganapathiraju SC, Streng PS and Smith DI: Long, abundantly expressed non-coding transcripts are altered in cancer. Hum Mol Genet 17: 642-655, 2008.

6. Mattick JS: The functional genomics of noncoding RNA. Science 309: 1527-1528, 2005.

7. Guttman M, Donaghey J, Carey BW, Garber M, Grenier JK, Munson G, Young G, Lucas AB, Ach R, Bruhn L, et al: lincRNAs act in the circuitry controlling pluripotency and differentiation. Nature 477: 295-300, 2011.

8. Rinn JL, Kertesz M, Wang JK, Squazzo SL, Xu X, Brugmann SA, Goodnough LH, Helms JA, Farnham PJ, Segal E and Chang HY: Functional demarcation of active and silent chromatin domains in human HOX loci by noncoding RNAs. Cell 129: 1311-1323, 2007.

9. Ponting CP, Oliver PL and Reik W: Evolution and functions of long noncoding RNAs. Cell 136: 629-641, 2009.

10. Hung T, Wang Y, Lin MF, Koegel AK, Kotake Y, Grant GD, Horlings HM, Shah N, Umbricht C, Wang P, et al: Extensive and coordinated transcription of noncoding RNAs within cell-cycle promoters. Nat Genet 43: 621-629, 2011.
11. Lanz RB, McKenna NJ, Onate SA, Albrecht U, Wong J, Tsai SY, Tsai MJ and O'Malley BW: A steroid receptor coactivator, SRA, functions as an RNA and is present in an SRC-1 complex. Cell 97: 17-27, 1999.

12. Ling H, Vincent K, Pichler M, Fodde R, Berindan-Neagoe I, Slack FJ and Calin GA: Junk DNA and the long non-coding RNA twist in cancer genetics. Oncogene 34: 5003-5011, 2015.

13. Leygue E: Steroid receptor RNA activator (SRA1): Unusual bifaceted gene products with suspected relevance to breast cancer. Nucl Recept Signal 5: e006, 2007.

14. Colley SM and Leedman PJ: Steroid receptor RNA activator-A nuclear receptor coregulator with multiple partners: Insights and challenges. Biochimie 93: 1966-1972, 2011.

15. Eoh KJ, Paek J, Kim SW, Kim HJ, Lee HY, Lee SK and Kim YT: Long non-coding RNA, steroid receptor RNA activator (SRA), induces tumor proliferation and invasion through the NOTCH pathway in cervical cancer cell lines. Oncol Rep 38: 3481-3488, 2017.

16. Carninci P, Kasukawa T, Katayama S, Gough J, Frith MC, Maeda N, Oyama R, Ravasi T, Lenhard B, Wells C, et al: The transcriptional landscape of the mammalian genome. Science 309: 1559-1563, 2005.

17. Hall PA and Russell SH: New perspectives on neoplasia and the RNA world. Hematol Oncol 23: 49-53, 2005.

18. Mercer TR, Dinger ME and Mattick JS: Long non-coding RNAs: Insights into functions. Nat Rev Genet 10: 155-159, 2009.

19. Kim HJ, Lee DW, Yim GW, Nam EJ, Kim S, Kim SW and Kim YT: Long non-coding RNA HOTAIR is associated with human cervical cancer progression. Int J Oncol 46: 521-530, 2015.

20. Gupta RA, Shah N, Wang KC, Kim J, Horlings HM, Wong DJ, Tsai MC, Hung T, Argani P, Rinn JL, et al: Long non-coding RNA HOTAIR reprograms chromatin state to promote cancer metastasis. Nature 464: 1071-1076, 2010.

21. Schiffman M, Castle PE, Jeronimo J, Rodriguez AC and Wacholder S: Human papillomavirus and cervical cancer. Lancet 370: 890-907, 2007.

22. Lui WO, Pourmand N, Patterson BK and Fire A: Patterns of known and novel small RNAs in human cervical cancer. Cancer Res 67: 6031-6043, 2007.

23. Fatima R, Akhade VS, Pal D and Rao SM: Long noncoding RNAs in development and cancer: Potential biomarkers and therapeutic targets. Mol Cell Ther 3: 5, 2015.

24. Lee MY and Shen MR: Epithelial-mesenchymal transition in cervical carcinoma. Am J Transl Res 4: 1-13, 2012.

25. Ramis-Conde I, Chaplain MA, Anderson AR and Drasdo D: Multi-scale modelling of cancer cell intravasation: The role of cadherins in metastasis. Phys Biol 6: 016008, 2009.

26. Calaf GM, Balajee AS, Montalvo-Villagra MT, Leon M, Daniela NM, Alvarez RG, Roy D, Narayan G and Abarca-Quinones J: Vimentin and Notch as biomarkers for breast cancer progression. Oncol Lett 7: 721-727, 2014.

27. Martin TA, Goyal A, Watkins G and Jiang WG: Expression of the transcription factors snail, slug, and twist and their clinical significance in human breast cancer. Ann Surg Oncol 12: 488-496, 2005.

28. Delgado G, Bundy B, Zaino R, Sevin BU, Creasman WT and Major F: Prospective surgical-pathological study of disease-free interval in patients with stage IB squamous cell carcinoma of the cervix: A Gynecologic Oncology Group study. Gynecol Oncol 38: 352-357, 1990.

29. Wentz WB and Reagan JW: Survival in cervical cancer with respect to cell type. Cancer 12: 384-388, 1959.

30. Biewenga P, van der Velden J, Mol BW, Stalpers LJ, Schilthuis MS, van der Steeg JW, Burger MP and Buist MR: Prognostic model for survival in patients with early stage cervical cancer. Cancer 117: 768-776, 2011.

31. Kodama J, Seki N, Masahiro S, Kusumoto T, Nakamura K, Hongo A and Hiramatsu Y: Prognostic factors in stage IB-IIB cervical adenocarcinoma patients treated with radical hysterectomy and pelvic lymphadenectomy. J Surg Oncol 101: 413-417, 2010 . 